\title{
Características socioeconômicas e demográficas do desemprego: a Região Metropolitana de Curitiba em tela
}

\author{
Luís Abel da Silva Filho* \\ Maria do Livramento Miranda Clementino**
}

\begin{abstract}
Resumo: O desemprego no Brasil mostrou-se acentuado durante o processo de abertura econômica e perdurou por todos os anos da década de 1990. Nos anos 2000, ainda se assistiu a elevadas estatísticas do desemprego bastante nítido no tecido metropolitano brasileiro, com maior ênfase nas áreas metropolitanas de economias menos dinâmicas. Ante isso, é pretensão deste artigo fazer uma análise do mercado de trabalho na Região Metropolitana de Curitiba, com ênfase no desemprego e seus componentes. Os dados utilizados são da Pesquisa Nacional por Amostra de Domicílios (PNAD), no recorte temporal compreendido entre os anos de 2001-2008. Metodologicamente recorre-se a uma breve revisão de literatura e posteriormente se utiliza o método de composição do desemprego. Os principais resultados encontrados evidenciam a redução do desemprego nessa RM ao longo dos anos. Porém, constatou-se a elevação da participação das mulheres, jovens e idosos, brancos e dos mais escolarizados no estoque total de desempregados na RM de Curitiba.
\end{abstract}

Palavras-chave: RM de Curitiba; Mercado de trabalho; Desemprego.

Classificação JEL: J2; J29; J82.

\footnotetext{
" Mestre em Economia pela Universidade Federal do Rio Grande do Norte (UFRN). Professor do Departamento de Economia da Universidade Regional do Cariri (URCA) e pesquisador do Observatório das Metrópoles da Universidade Federal do Rio Grande do Norte (UFRN). Endereço eletrônico: abeleconomia@hotmail.com.

${ }^{* *}$ Doutora em Economia pela Universidade Estadual de Campinas (UNICAMP). Professora titular do Departamento de Políticas Públicas da Universidade Federal do Rio Grande do Norte (UFRN) e coordenadora de rede do Observatório das Metrópoles, núcleo da UFRN. Endereço eletrônico: clement@ufrnet.br.
} 


\section{Considerações iniciais}

O desemprego metropolitano atingiu elevados índices nos anos de 1990 e adentraram os anos 2000 com fortes impactos nas grandes metrópoles brasileiras. A globalização da economia e seus impactos com as novas formas de produção foram fatores determinantes da elevação do desemprego aberto nos países em desenvolvimento. $\mathrm{O}$ tecido metropolitano não ficou incólume aos modelos de produção pautados em elevadas tecnologias, impondo-se como consequência a redução de força de trabalho no processo produtivo.

O modelo de produção incorporado pela acumulação flexível possibilitou ganhos de escopo e de escala, concomitantemente à redução do contingente de trabalhadores utilizados no processo (Santos, Moreira, 2006; Silva Filho, Queiroz, 2010). A globalização econômica foi, segundo Beck (1999), fator determinante da expansão do "capitalismo sem trabalho" por todo o mundo. A partir desse fenômeno, as elevadas taxas de desemprego se fizeram sentir nos países menos desenvolvidos, nos quais ainda é elevado o contingente de trabalhadores com baixos níveis de instrução, sendo esta fator determinante do emprego no capitalismo avançado.

O desemprego metropolitano foi sobremaneira acentuado, dada a forte concentração produtiva nessas áreas mais dinâmicas. Além disso, os impactos da tecnologia de ponta utilizada no processo produtivo ficaram concentrados nessas áreas em razão dos fatores já citados. Ademais, as áreas metropolitanas brasileiras concentram forte contingente populacional que migram do interior do país em busca de empregos. Em muitos casos, mão de obra sem instrução ou com baixo nível educacional, que procura trabalho nas grandes cidades e acaba entrando nas estatísticas do desemprego.

As transformações econômicas mundiais do final do século XX e início do XXI não deixaram incólume nenhuma área metropolitana brasileira. $\mathrm{O}$ desemprego elevado foi registrado em todas elas, com elevados índices para os grupos sociais mais vulneráveis (Bastos, 2010). Dentre eles, jovens, idosos, não brancos e mulheres (Silva Filho, 2011). Mesmo as áreas de maior dinamismo econômico do país, notadamente nas metrópoles do eixo sul-sudeste, padeceram em elevada escala a falta de emprego.

Destarte, é pretensão deste artigo avaliar o perfil do desemprego 
metropolitano na RM de Curitiba. Mesmo sendo ela uma das RM com melhores indicadores de qualidade de vida, faz-se necessário observar se o desemprego também é seletista. Os dados utilizados referem-se aos anos de 2001-2008, da primeira década, portanto, do século corrente. A fonte de dados é a Pesquisa Nacional por Amostra de Domicílios (PNAD). Eles foram trabalhados mediante utilização do método de composição do desemprego proposto por Corseuil et al. (1997) e replicado por Bastos (2010) e Silva Filho e Amon-Há (2011), identificando assim as principais características dele no tecido metropolitano da grande Curitiba.

Para atingir o objetivo proposto, o artigo está estruturado da forma que se segue: além dessas considerações iniciais, a segunda seção aborda algumas características do mercado de trabalho da RM de Curitiba, no final do século XX e início do XXI; a terceira seção apresenta os procedimentos metodológicos aqui adotados; a quarta seção descreve e analisa os principais resultados encontrados pelo estudo; e, por último, traçam-se algumas considerações finais.

\section{Mercado de trabalho na Região Metropolitana de Curitiba: algumas considerações}

O mercado de trabalho da RM de Curitiba tem apresentado relativo desempenho na comparação com as demais regiões metropolitanas brasileiras. A taxa de atividade era a mais elevada, segundo dados da PNAD e da PME, até 2007. Considera-se ainda elevada a participação dos vínculos formais de trabalho na RM (Maranho, 2008; Garcias, Lyra, 2002; Sampaio, 2005; Silva, 2005).

A dinâmica econômica regional está diretamente associada a reduzidas taxas de desemprego e à contratação de postos de trabalho com vínculos formais (Maranho, 2008). A indústria automobilística da RM de Curitiba tem elevada importância na dinâmica local de seu mercado de trabalho, bem como nas suas contratações pós-instalações. Além disso, a concentração de atividades produtivas na RM tem relativo sucesso na redução do desemprego metropolitano ao longo dos anos.

Em relação à formalidade do mercado de trabalho da RM de Curitiba, os dados postados no Gráfico 1, a partir da informações da PNAD (2001-2008), 
revelam o bom desempenho registrado no período em análise. Cabe destacar que tais resultados não são exclusivos dessa RM, mas decorrem do bom desempenho vivido pela economia brasileira a partir de 2004. A recuperação econômica do país a partir de então tem refletido melhor desempenho no mercado de trabalho nacional e elevado as taxas de contratações de trabalhadores com registros em carteira de trabalho (Dedecca, Rosandiski, 2006; Remy et al., 2010; Silva Filho, Queiroz, 2011).

É pertinente, portanto, destacar que a RM de Curitiba apresenta bons resultados conforme observado no estudo de Silva Filho (2011). Nesse estudo, ficou constatado que nas três RM do Nordeste - Fortaleza, Recife e Salvador -, a informalidade é sobremaneira acentuada, representando 43,2\%, 50,0\% e 51,4\%, no ano de 2008, respectivamente. Com isso, pode-se interpretar, conforme Garcias e Lyra (2002), que o nível de desenvolvimento regional é crucial para manutenção das taxas de formalidade no mercado de trabalho, assim como a taxa de atividade da PEA.

O bom desempenho da economia nacional não pode deixar de ser considerado. Os dados mostram que nos anos de 2001 e 2002, o primeiro pela crise energética do país e o segundo pela interferência das incertezas acerca da política nacional (Remy et al, 2010; Maranho, 2008), registraram-se as mais elevadas taxas de informalidade no mercado de trabalho metropolitano de Curitiba.

Em 2003, ano em que se iniciam os primeiros sinais de recuperação da economia brasileira traduzidos na elevação das taxas de crescimento do Produto Interno Bruto (PIB) (Dedecca, Rosandiski, 2006; Silva Filho, Queiroz, 2011), eleva-se a participação da formalidade no mercado de trabalho da RM de Curitiba, ao se registrarem $60,3 \%$ dos postos de trabalhos protegidos pela Consolidação das Leis do Trabalho (CLT). 
Gráfico 1 - Percentual da PEA ocupada segundo a proteção no trabalho na Região Metropolitana de Curitiba (2001-2008)

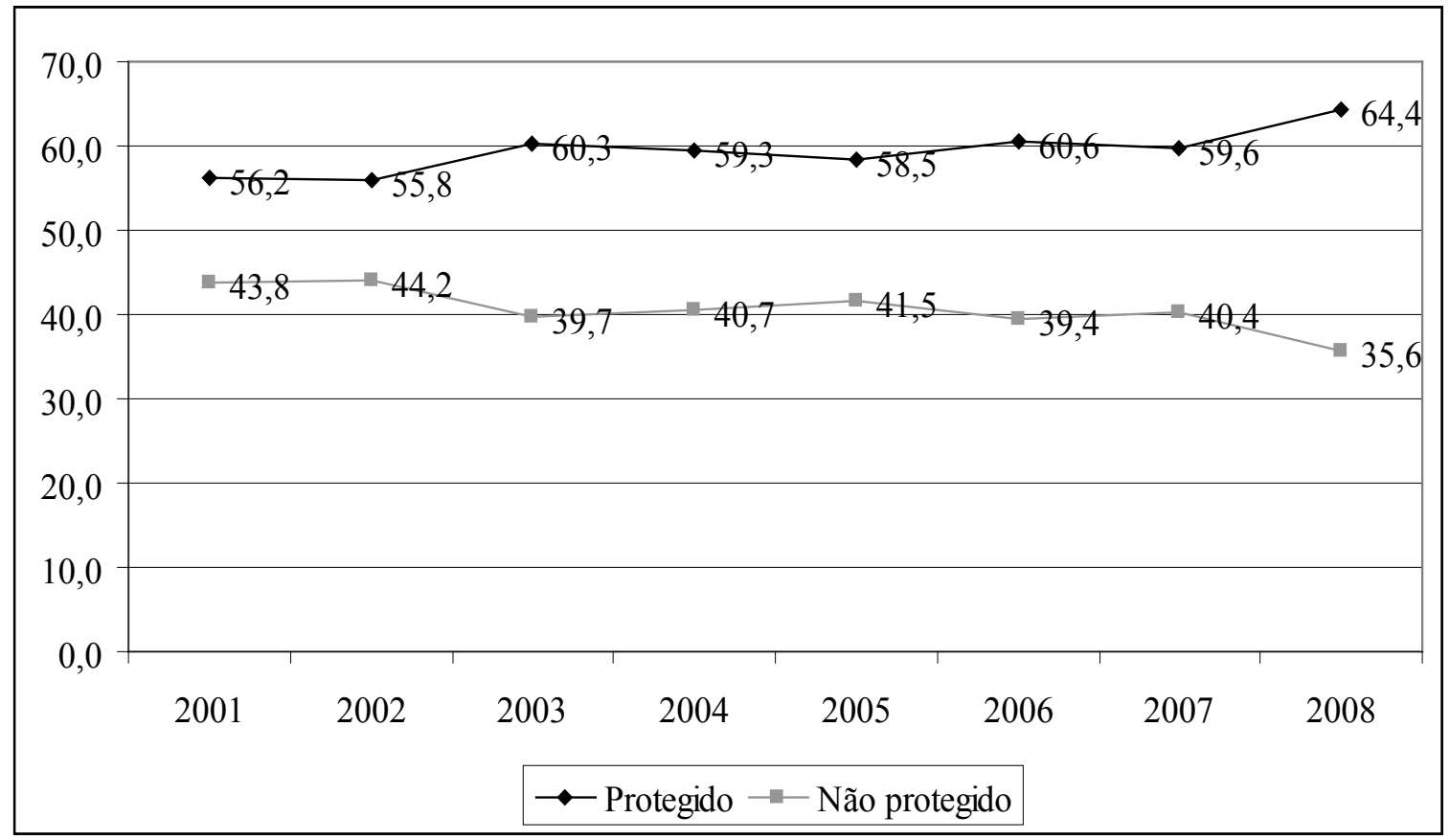

Fonte: PNAD, 2001-2008; IBGE.

A partir de 2003, elevam-se os vínculos formais de trabalho, embora com oscilação ora positiva ora negativa. Ressalte-se, porém, que eles permanecem em patamares superiores aos observados nos dois primeiros anos da década. Além disso, em 2008, mesmo em um contexto de crise econômica internacional, a RM de Curitiba registra sua maior taxa de formalidade entre os anos aqui observados, com $64,4 \%$ de sua PEA ocupada protegida no trabalho.

Em relação ao desemprego, objeto central deste estudo, observa-se no Gráfico 2 a tendência de redução ao longo dos anos analisados. Entre 2001 e 2004, registram-se, segundo a PNAD, oscilações destacáveis do fenômeno. No primeiro ano, o desemprego metropolitano em Curitiba chega a atingir 9,4\% da PEA, reduzindo-se levemente para 9,1\% no segundo ano. Em 2003 registra-se a mesma taxa observada em 2001, mas, já em 2004, ano em que se efetiva, de fato, melhora na performance macroeconômica nacional, pautada pelo maior desempenho das exportações do país (Cintra, 2005), observa-se redução no desemprego para 8,1\%, menor resultado observado até então. 
Gráfico 2 - Taxa de desemprego aberto na

Região Metropolitana de Curitiba (2001-2008)

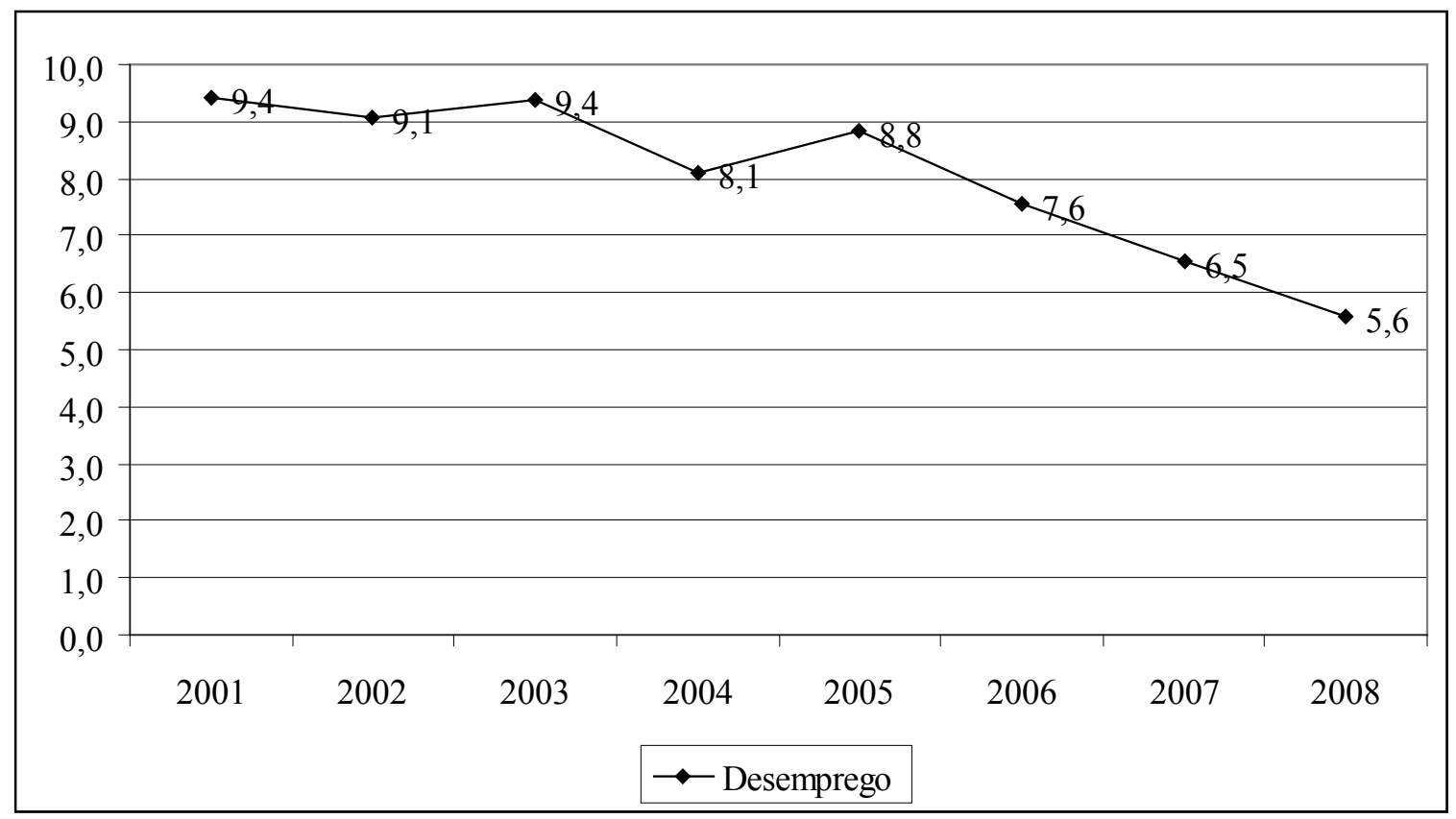

Fonte: PNAD, 2001-2008; IBGE.

A partir de 2005, o desemprego metropolitano em Curitiba segue trajetória de redução constante. Nesse ano, registra-se 8,8\% da PEA metropolitana desempregada, reduzindo-se para 5,6\% em 2008. Destaque-se que, mesmo diante de um contexto de crise no mercado externo, o desemprego na RM de Curitiba permaneceu em baixos índices naquele ano. As políticas de intervenção do Governo Federal para o setor automobilístico, de forte representatividade na RM de Curitiba, podem ter reduzido o impacto do desemprego no mercado de trabalho local.

A partir das informações aqui expostas, a seção que se segue traça os procedimentos metodológicos utilizados na pesquisa, para que se possa inferir sobre as características do desemprego metropolitano em Curitiba, segundo os grupos sociais.

\section{Procedimentos metodológicos}

O presente artigo toma como área de abrangência a Região Metropolitana de Curitiba-PR, que, segundo o IBGE, contava com população de 3.260.292 habitantes no ano de 2008, distribuída entre 26 municípios. Além disso, ela é a segunda maior região metropolitana do sul do Brasil e a oitava do país. A 
densidade demográfica é de 205,53 habitantes por km² (IBGE, 2011).

Segundo a PNAD, em 2008 a RM de Curitiba contava com 2.761.791 pessoas em idade ativa, com 1.799 .772 economicamente ativos; 65,17\% da PIA estavam na PEA, com 94,42\% da PEA ocupada. Acrescente-se ainda que somente $35,60 \%$ deles tinham vínculos formais de trabalho, sendo, portanto, a maioria na informalidade. Mesmo que somente 5,60\% da PEA estivesse desempregada em 2008, esse é nosso objeto de estudo.

Além da área de estudo já apresentada, o método utilizado foi empregado por Corseuil et al (1997) em estudo que investigou as metrópoles brasileiras, no período que compreende os anos de 1986 e 1995, utilizando-se dados da Pesquisa Mensal de Emprego (PME) e do Instituto Brasileiro de Geografia e Estatística (IBGE). Recentemente, Bastos (2010) empregou tal método para observar, em anos mais recentes, as metrópoles brasileiras e o Distrito Federal, na série temporal entre 1999 e 2007, com dados da Pesquisa de Emprego e Desemprego (PED) do DIEESE/SEADE.

Aqui, os dados são provenientes da Pesquisa Nacional por Amostra de Domicílios (PNAD) do IBGE. Os anos compreendidos estão entre 2001-2008 e as variáveis utilizadas são as que se seguem:

- Estoque total de desempregados na Região Metropolitana de Curitiba: tratase da População Economicamente Ativa que se declarou desempregada na semana de referência da pesquisa;

- Estoque de desempregados segundo o sexo: masculino e feminino;

- Estoque de desempregados segundo a raça/cor: branco e não branco, sendo que os não brancos são compostos por pretos e pardos;

- Estoque de desempregados segundo a faixa etária: 10 a 14; 15 a 24; 25 a 39; 40 a 59 ; 60 ou mais anos;

- Estoque de desempregados segundo os anos de estudo: 0 a 4; 5 a 8; 9 a 11; 12 ou mais anos.

A partir daí, pode-se classificar o estoque de desempregados da RMC da forma que se segue: 


$$
U_{i} / U=U_{i} / N_{i} \cdot N_{i} / P_{i} \cdot P_{i} / P \cdot P / N \cdot N / U
$$

em que, $U=$ estoque total de desempregados na Região Metropolitana de Curitiba (2001-2008); $U_{i}=$ assume o caráter de estoque de desempregados do grupo $i$ na RMC; $N=$ número de membros da PEA total na RMC; $N_{i}=$ número de membros da PEA do grupo $i$ na RMC; $P=$ número de membros da PIA total na RMC; $P_{i}=$ número de membros da PIA do grupo $i$.

A Equação 1, pode ser reescrita da seguinte forma:

$$
U_{i} / U=\frac{U_{i} / N_{i}}{U / N} \cdot \frac{N_{i} / P_{i}}{N / P} \cdot \frac{P_{i}}{P}
$$

Transformando em logaritmos os dados da Equação (2), pode-se captar o efeito elasticidade na decomposição dos fatores que afetam o desemprego na RMC.

Dessa forma, tem-se:

$$
L_{n}\left(U_{i} / U\right)=\left[\operatorname{Ln}\left(U_{i} / N_{i}\right)-L_{n}(U / N)\right]+\left[L_{n}\left(N_{i} / P_{i}\right)-L_{n}(N / P)\right]+L_{n}\left(P_{i} / P\right)
$$

Com esta expressão, temos as variações expressas da forma que segue:

$$
\Delta L_{n}\left(U_{i} / U\right)=\left[\Delta L_{n}\left(U_{i} / N_{i}\right)-\Delta L_{n}(U / N)\right]+\left[\Delta L_{n}\left(N_{i} / P_{i}\right)-\Delta L_{n}(N / P)\right]+\Delta L_{n}\left(P_{i} / P\right)
$$

Conforme proposto por Corseuil et al. (1997), e empregado por Bastos (2010), a variação da participação do grupo $i$ no estoque de desempregados numa região pode ser captado por sua variação em relação à variação da região. Com isso, a participação dos desempregados de um determinado grupo $i$ em uma região está relacionada à variação do peso deste grupo populacional na PIA da região em observação; a taxa de participação da força de trabalho do grupo $i$ na taxa da participação da força de trabalho da região; e a participação da taxa de desempregos $i$ em relação à taxa total de desempregados existentes na região.

A partir desses esclarecimentos, obtêm-se os resultados encontrados na RMC na seção que se segue. 


\section{Resultados encontrados}

A partir dos dados plotados na Tabela 1, é possível observar que houve redução da participação dos homens no estoque total de desempregados na RM de Curitiba entre 2001-2008. Para tanto, contribuíram o comportamento da taxa de participação da força de trabalho do grupo em comparação com o da força de trabalho da região, assim como a variação do peso do grupo em comparação com o da PIA da região.

Tabela 1 - Evolução da participação segundo o sexo no desemprego

\begin{tabular}{lcc}
\multicolumn{4}{c}{ e seus componentes - RM de Curitiba $(2001-2008)$} \\
\hline \multicolumn{1}{c}{ Descrição } & Masculino & Feminino \\
\hline$\Delta l_{n}\left(U_{i} / U\right)$ & $-0,0208$ & 0,0161 \\
$\Delta l_{n}\left(U_{i} / N_{i}\right)-\Delta l_{n}(U / N)$ & 0,0472 & $-0,0687$ \\
$\Delta l_{n}\left(N_{i} / P_{i}\right)-\Delta l_{n}(N / P)$ & $-0,0267$ & 0,0462 \\
$\Delta L_{n}\left(P_{i} / P\right)$ & $-0,0413$ & 0,0386 \\
\hline
\end{tabular}

Fonte: PND, 2001-2008; IBGE.

Contrariamente ao observado para a força de trabalho masculina, elevouse a participação da força de trabalho feminina no estoque total de desempregados na RM Curitiba. Nesse caso, tanto a variação do peso do grupo em comparação com a PIA da região quanto o comportamento da taxa de participação da força de trabalho do grupo em comparação com o da força de trabalho da região corroboram no sentido de levar as mulheres ao desemprego. Em outras palavras, tanto aumentou a participação da PIA feminina proporcionalmente a PIA da RM de Curitiba quanto se elevou a participação destas no mercado de trabalho em busca de emprego.

Já em relação à raça/cor, cresceu a participação da força de trabalho que se declarou branca no desemprego metropolitano de Curitiba. O fator que contribuiu acentuadamente para esse resultado foi o comportamento da taxa de desemprego do grupo em comparação ao da taxa de desemprego da região. Os demais fatores variaram de forma a retirá-los do desemprego, sendo que em menor proporção que o fator citado, o que não conseguiu reverter o quadro desse grupo no desemprego metropolitano. 
Tabela 2 - Evolução da participação segundo a raça/cor no desemprego e seus componentes - RM de Curitiba (2001-2008)

\begin{tabular}{lr|c}
\multicolumn{1}{c|}{ Descrição } & Branco & Não branco \\
\hline$\Delta l_{n}\left(U_{i} / U\right)$ & 0,0141 & $-0,0719$ \\
$\Delta l_{n}\left(U_{i} / N_{i}\right)-\Delta l_{n}(U / N)$ & 0,0806 & $-0,3353$ \\
$\Delta l_{n}\left(N_{i} / P_{i}\right)-\Delta l_{n}(N / P)$ & $-0,0006$ & $-0,0053$ \\
$\Delta L_{n}\left(P_{i} / P\right)$ & $-0,0658$ & 0,2688 \\
\hline
\end{tabular}

Fonte: PNAD, 2001-2008; IBGE.

Nota: Não branco corresponde a pretos e pardos.

Para os não brancos, deu-se o movimento contrário. Tanto o comportamento da taxa de desemprego do grupo em comparação com o da taxa de desemprego da região quanto o comportamento da taxa de participação na força de trabalho do grupo em comparação com o da força de trabalho da região corroboraram o resultado. Para eles, apenas a variável demográfica - traduzida como a variação do peso do grupo em relação à PIA da região - teve influência para levá-los ao desemprego, o que não foi suficiente para atingir tal resultado.

Considerando-se a PEA desempregada segundo a faixa etária, os dados da Tabela 3 apresentam a tendência da variável idade como forte determinante do desemprego metropolitano em Curitiba. Nesse caso, com exceção da faixa etária entre 15 a 24 anos, todas as demais foram elevadas no estoque total de desempregados. Conforme se observa, cresceu a participação daqueles com idade entre 10 e 14 anos no desemprego da RM de Curitiba.

$\mathrm{Na}$ faixa etária seguinte (15 a 24 anos), ocorreu redução desses no estoque total de desempregados. $\mathrm{O}$ fator que mais corroborou o resultado foi a variação do peso do grupo na PIA da região (-0,2237). Além desse, registrou-se ainda o comportamento da taxa de participação da força de trabalho do grupo em comparação com o da taxa de participação na força de trabalho da região $(-0,0172)$.

Tabela 3 - Evolução da participação segundo a faixa etária no desemprego e seus componentes - RM de Curitiba (2001-2008)

\begin{tabular}{l|c|c|c|c|c}
\hline \multicolumn{1}{c|}{ Descrição } & De 10 a 14 & De 15 a 24 & De 25 a 39 & De 40 a 59 & 60 ou mais \\
\hline$\Delta l_{n}\left(U_{i} / U\right)$ & 0,3194 & $-0,0614$ & 0,0556 & 0,0268 & 0,1653 \\
$\Delta l_{n}\left(U_{i} / N_{i}\right)-\Delta l_{n}(U / N)$ & 0,8517 & 0,1794 & 0,0324 & $-0,1147$ & 0,0298 \\
$\Delta l_{n}^{n}\left(N_{i} / P\right)-\Delta l_{n}^{n}(N / P)$ & $-0,6306$ & $-0,0172$ & 0,0299 & 0,0234 & 0,0510 \\
$\Delta L_{n}\left(P_{i} / P\right)$ & 0,0983 & $-0,2237$ & $-0,0068$ & 0,1181 & 0,0845 \\
\hline
\end{tabular}

Fonte: PNAD, 2001-2008; IBGE.

Já na faixa etária de 25 a 24 anos somente a variação do peso do grupo na PIA da região foi favorável a retirá-los do desemprego, o que não foi suficiente 
para reduzir o estoque de desempregados do grupo em relação ao estoque total de desempregados. Além deles, a PEA desocupada com idade entre 40 e 59 anos contou apenas com o comportamento da taxa de desemprego do grupo em comparação ao da taxa de desemprego da região $(-0,1147)$, o que também não bastou para retirá-los do estoque total de desempregados.

Para a PEA desocupada com 60 anos ou mais de idade, todos os fatores corroboraram para elevar sua participação no estoque total de desempregados. Porém, o que mais contribuiu foi a variação do peso do grupo na PIA da região, ou seja, aumentou a participação de pessoas idosas na PIA da RM de Curitiba, o que pode ser atribuído à elevação na longevidade do brasileiro assistida nos últimos anos, sobretudo nessa RM onde se registram os mais elevados indicadores de qualidade de vida.

Quando observados os anos de escolaridade da PEA desocupada na RM de Curitiba, os dados da Tabela 4 mostram contraste com o esperado nesse estudo. A PEA desocupada com escolaridade entre 0 e 4 anos de estudos sofreu redução acentuada em sua participação no estoque total de desempregados. Além deles, aqueles com escolaridade entre 5 e 8 anos de estudos também se reduziram, só que em menor proporção. Nesse caso, tanto para o primeiro grupo quanto para o segundo, todos os fatores corroboraram no sentido de retirá-los do desemprego.

Tabela 4 - Evolução da participação segundo os anos de estudo no desemprego e seus componentes - RM de Curitiba (2001-2008)

\begin{tabular}{l|r|r|r|c}
\hline \multicolumn{1}{c|}{ Descrição } & De 0 a 4 & De 5 a 8 & De 9 a 11 & 12 ou mais \\
\hline$\Delta l_{n}\left(U_{i} / U\right)$ & $-0,6218$ & $-0,2870$ & 0,3216 & 0,5373 \\
$\Delta l_{n}\left(U_{i} / N_{i}\right)-\Delta l_{n}(U / N)$ & $-0,1367$ & $-0,1242$ & 0,0991 & 0,1770 \\
$\Delta l_{n}\left(N_{i} / P_{i}\right)-\Delta l_{n}(N / P)$ & $-0,1583$ & $-0,0872$ & 0,0029 & 0,0135 \\
$\Delta L_{n}\left(P_{i} / P\right)$ & $-0,3267$ & $-0,0756$ & 0,2195 & 0,3469 \\
\hline
\end{tabular}

Fonte: PNAD, 2001-2008; IBGE.

Porém, para a PEA desocupada com escolaridade entre 9 e 11 anos e com 12 anos ou mais de estudo, elevou-se sua participação no estoque total de desempregados. Por trás disso tudo, podem estar implícitos os efeitos da reestruturação produtiva e a alavancagem da economia nacional com a criação de postos de trabalho acentuadamente precários. Nesse caso, a força de trabalho com mais anos de estudo passa mais tempo em busca de bons postos de trabalho ou não aceitam qualquer tipo de emprego. Além do mais, elevou-se a escolaridade 
dos brasileiros nos últimos anos, o que torna mais competitivas as vagas que surgem no mercado de trabalho, sem esquecermos que mais anos de estudo no país não significam necessariamente garantia de postos de trabalho.

Destaque-se ainda que todos os fatores contribuíram para elevar a PEA desocupada com mais anos de estudos no estoque total de desempregados na RM Curitiba. Ou seja, pode-se aceitar que há maior competitividade para a força de trabalho mais escolarizada e maior vulnerabilidade dela no desemprego metropolitano.

\section{Considerações finais}

O objetivo deste artigo foi observar o mercado de trabalho e a composição do desemprego na RM de Curitiba. Os principais resultados encontrados mostram bom desempenho do mercado de trabalho metropolitano ao longo dos anos analisados.

Os registros da PNAD entre 2001-2008 revelam elevação na participação dos ocupados com registros formais de trabalho. Em 2001 eles correspondiam a $56,2 \%$ dos ocupados, elevando-se para 64,4\% em 2008. Além disso, o desemprego metropolitano reduziu-se de $9,4 \%$ no primeiro para $5,6 \%$ no último ano analisado.

Em relação à composição do desemprego, assistiu-se à elevação da PEA desocupada feminina no estoque total de desempregados, contrariamente à observada para a masculina, que se mostrou reduzida no período (2001-2008). Adicionalmente, cresceu a participação da PEA desocupada de raça/cor branca no estoque total de desempregados e registrou-se redução dos não brancos na composição do estoque de desempregados na RM de Curitiba nos mesmos anos.

Em relação ao desemprego por idade, somente a PEA desocupada na faixa etária de 15 a 24 anos apresentou redução no estoque total de desempregados. Além disso, todos os fatores corroboraram a elevação daqueles com 60 anos ou mais de idade no estoque total de desempregados na RM de Curitiba entre 20012008.

Observou-se ainda a redução da força de trabalho com menos anos de estudo no estoque total de desempregados (primeira e segunda faixa), havendo 
todos os fatores contribuído para esse fim, e a elevação da PEA desocupada com mais anos de estudos (segunda e terceira faixa) no desemprego metropolitano em Curitiba, cabendo destacar também que todos os fatores contribuíram para o resultado encontrado.

Diante disso, pode-se assegurar bom desempenho do mercado de trabalho da RM de Curitiba nos últimos anos. Porém, cabe acrescentar que o desemprego é seletista e atinge grupos vulneráveis, tais como, mulheres, brancos, muito jovens e idosos, além de se ter elevado, curiosamente, a participação dos mais escolarizados no estoque total de desempregados entre os anos aqui observados.

\section{Referências}

Bastos, R. L. A. (2010). "Desemprego metropolitano no Brasil -1999-2007”. Anais do Encontro Nacional da Associação Brasileira de Estudos Populacionais (ABEP).

Beck, Ulrich. (1999). O que é globalização? Equívocos do globalismo respostas à globalização. São Paulo: Paz e Terra.

Cintra, Marcos Antonio Macedo. (2005). "Suave fracasso - a política macroeconômica brasileira entre 1999 e 2005”. Revista Novos Estudos, novembro de 2005.

Corseuil, C. et al. (1997). "Determinantes da evolução da estrutura do desemprego no Brasil: 1986-1995”. Economia Aplicada 1(3): 443-467.

Dedecca, Claudio Salvadori; Rosandiski, Eliane Navaro. (2006). "Recuperação econômica e geração de empregos formais". Revista Parcerias Estratégicas 22.

Garcias, P. M.; Lyra, S. A. (2002). "Mercado de trabalho na região metropolitana de Curitiba”. Nota técnica IPARDES.

Instituto Brasileiro de Geografia e Estatística. Pesquisa Nacional por Amostra de Domicílios - PNAD 2001 2008: Pesquisa Básica.

Maranho, E. J. (2008). "Principais tendências do mercado de trabalho da região metropolitana de Curitiba - RMC - 2003 a 2007”. Anais do Encontro Nacional da Associação Brasileira de Estudos populacionais (ABEP).

Remy, M. A. P. de A.; Queiroz, S. N. de; Silva Filho, L. A. da. (2010). "Evolução recente do emprego formal no Brasil - 2000-2008. Anais do Encontro Nacional da Associação Brasileira de Estudos Populacionais (ABEP).

Sampaio, A. V. (2005). "O mercado de trabalho no estado do Paraná entre 1992 e 2003”. IPEA mercado de trabalho 27.

Santos, L. M. dos; Moreira, I. T. (2006). "Condições do mercado de trabalho no setor de serviços no nordeste". Anais do Encontro Nacional de Estudos Populacionais (ABEP).

Silva, C. C. (2005). "A dinâmica do mercado de trabalho na região metropolitana de 
Curitiba”. IPEA mercado de trabalho 27.

Silva Filho, L. A.; Queiroz, S. N. de. (2010). "Evolução das formas de produção e do trabalho: uma abordagem do marxismo ao toyotismo". Revista de Economia Política e Historia Econômica 21: 55-86.

Silva Filho, L. A.; Queiroz, S. N. de. (2011). "Recuperação econômica e emprego formal: avaliação empírica para o nordeste brasileiro - 2000/2008”. Revista Perspectiva Econômica 7(1): 42-54.

Silva Filho, L. A. (2011). Mercado de trabalho e estrutura sócio-ocupacional: estudo comparativo entre as regiões metropolitanas de Fortaleza, Recife e Salvador - 2001-2008. Natal: dissertação de mestrado em Economia, Universidade Federal do Rio Grande do Norte.

Silva Filho, L. A.; Amon-Há, R. (2011). "Perfil do desemprego recente na Região Metropolitana de Salvador - 2001-2008”. VII Encontro de Economia Baiana. 